\title{
MicroRNA-34b has an oncogenic role in esophageal squamous cell carcinoma
}

\author{
KOSHIRO HARATA, HIDEYUKI ISHIGURO, YOSHIYUKI KUWABARA, MASAHIRO KIMURA, AKIRA MITSUI, \\ RYO OGAWA, TAKEYASU KATADA, TATSUYA TANAKA, MIDORI SHIOZAKI and YOSHITAKA FUJII
}

Nagoya City University Graduate School of Medical Sciences, Oncology, Immunology and Surgery, Nagoya 467-8601, Japan

Received February 23, 2010; Accepted May 5, 2010

DOI: 10.3892/ol_00000120

\begin{abstract}
Esophageal squamous cell carcinoma (ESCC) is a common malignancy and one of the more difficult diseases to diagnose in Japan due to its poor prognosis. MicroRNAs are small non-coding RNAs of 21-23 nucleotides that regulate gene expression. MicroRNA-34b (miR-34b) has been reported to be overexpressed in various types of cancer. However, its role in ESCC has yet to be extensively studied. The present study investigated the expression of miR-34b in 88 ESCC patients. The miR-34b expression in ESCC was significantly higher than that in the corresponding normal esophageal mucosa. It was more highly expressed in tumors with more advanced stages. However, its expression did not correlate with the p53 status. Transfection of anti-miR-34b to the ESCC cells suppressed cell growth in vitro. These results suggest an oncogenic role of miR in ESCC.
\end{abstract}

\section{Introduction}

Esophageal squamous cell carcinoma (ESCC) is the ninth most frequent cancer and the sixth most frequent cause of death from malignant tumors in Japan. The number of deaths due to esophageal cancer has increased steadily. ESCC is often diagnosed at an advanced stage, although even in early stage a number of patients develop local tumor recurrence or distant metastasis within a short period after curative surgery. Although pre-operative chemotherapy and chemoradiotherapy are currently used for patients with advanced-stage ESCC, the effects of such modalities are unsatisfactory, prompting a search for new treatment strategies.

MicroRNAs (miRNAs) are a species of small non-coding single-stranded RNA of approximately 21-23 nucleotides that post-transcriptionally modulate gene expression by negatively

Correspondence to: Dr Hideyuki Ishiguro, Nagoya City University Graduate School of Medical Sciences, Oncology, Immunology and Surgery, 1 Kawasumi, Mizuho-cho, Mizuho-ku, Nagoya 467-8601, Japan

E-mail: h-ishi@med.nagoya-cu.ac.jp

Key words: microRNA-34b, esophageal squamous cell carcinoma, MTT assay regulating the stability or translational efficiency of their target mRNAs. miRNAs are a class of gene products that were recently found to play a role in several types of cancer (1-4). miRNAs may function as potent regulators of gene expression and altered miRNA levels result in aberrant expression of gene products that may contribute to cancer biology (5). Furthermore, certain miRNAs may function as either oncogenes or tumor-suppressor genes (6).

A previous study investigated the expression of 73 mature miRNAs in 30 ESCC patients using Taq Man quantitative PCR (7). The expression of miR-34b was high in all of the ESCC tissues. This study investigated miR-34b expression in 88 patients with ESCC and evaluated its correlation with clinicopathological features and postoperative survival. The miR-34b expression in ESCC cell lines was also examined and the effects of suppressing or overexpressing miR-34b on the proliferation of ESCC cells were analyzed.

\section{Materials and methods}

Patients and tumor samples. Esophageal cancer samples were obtained from 88 patients who had undergone surgery at the Nagoya City University Hospital, Japan, between January 1996 and December 2002. The study design was approved by the Institutional Review Board of our university hospital, and a written consent was obtained from all of the patients. Tumors were classified according to the Guidelines for the Clinical and Pathological Studies on Carcinoma of the Esophagus. The tumor and corresponding normal tissue were also obtained. Normal esophageal mucosa was removed from the apparently non-cancerous mucosa as far as possible from the tumor. The samples were frozen immediately in liquid nitrogen and stored at $-80^{\circ} \mathrm{C}$ until use. The characteristics of the 88 patients with ESCC are shown in Table I.

Cell lines and cell culture. ESCC cell lines (TE1-15) were obtained from the Japanese Collection of Research Bioresources. Cultures were maintained in RPMI-1640 (Sigma) supplemented with $10 \%$ fetal bovine serum (FBS) (Gibco) at $37^{\circ} \mathrm{C}$ in a humidified $5 \% \mathrm{CO}_{2}$ incubator.

A human esophageal squamous epithelial cell line (Het-1A) was obtained from the American Type Culture Collection. Het-1A was maintained in serum-free medium (LHC-9; BioSource) at $37^{\circ} \mathrm{C}$ in a humidified $5 \% \mathrm{CO}_{2}$ incubator. 
RNA extraction. Total RNA was extracted from ESCC tissue, as well as its corresponding non-cancerous mucosa, using the Absolutely RNA ${ }^{\mathrm{TM}}$ RT-PCR Miniprep kit (Stratagene, La Jolla, CA, USA) according to the manufacturer's instructions. The concentration of total RNA was adjusted to $2 \mathrm{ng} / \mu \mathrm{l}$ using a spectrophotometer.

Quantitative reverse transcription-polymerase chain reaction. Taq Man miRNA assays (ABI PRISM, Forest City, CA, USA) employed the stemloop method to detect the expression level of mature miR-34b. For reverse transcription (RT) reactions, $10 \mathrm{ng}$ of total RNA was used in each reaction (5 $\mu \mathrm{l})$ and mixed with the RT primer $(3 \mu \mathrm{l})$. The $\mathrm{RT}$ reaction was carried out at $16^{\circ} \mathrm{C}$ for $30 \mathrm{~min} ; 42^{\circ} \mathrm{C}$ for $30 \mathrm{~min} ; 85^{\circ} \mathrm{C}$ for $5 \mathrm{~min}$; and then maintained at $4^{\circ} \mathrm{C}$. After the RT reaction, $1.33 \mu \mathrm{l}$ of the cDNA was used for the polymerase chain reaction (PCR) along with Taq Man primers $(2 \mu \mathrm{l})$. The PCR reaction was conducted at $95^{\circ} \mathrm{C}$ for $10 \mathrm{~min}$ followed by 40 cycles of $95^{\circ} \mathrm{C}$ for $15 \mathrm{sec}$ and $60^{\circ} \mathrm{C}$ for $60 \mathrm{sec}$ in the ABI 7500 real-time PCR system. The real-time PCR results were analyzed and expressed as a relative miRNA expression of threshold cycle (CT) value. RT and PCR primers for miR-34b were purchased from ABI PRISM. The expression of miR-34b was calculated using the $2^{-\Delta \Delta C t}$ analysis method (8). The expression of miR-34b was normalized to that of the U6B small nuclear RNA gene (RNU6B).

p53 evaluation by immunohistochemistry (IHC). Immunohistochemical staining of p53 was performed on 4-mm sections from the paraffin-embedded tumors. Antigen retrieval was carried out by microwaving the tissue sections in phosphatebuffered saline (PBS) (0.1 M, pH 7.2) for $15 \mathrm{~min}$ followed by incubation with a monoclonal anti-p53 antibody ( $\mathrm{p}-53$ protein PAb240; Dako, Glostrup, Denmark) diluted 1:75 in PBS overnight at $4^{\circ} \mathrm{C}$. Primary antibody was detected by EnVision ${ }^{\mathrm{TM}} /$ HRP Mouse code K4001 (Dako) and visualized by Dako Liquid DAB. Counter staining was performed with Mayers hematoxylin. Nuclear p53 staining intensity was defined as high or low. Immunoreactivity for p53 was evaluated semiquantitatively by two observers and all tumors showing p53 immunoreactivity were assumed to have p53 mutation.

Transfection. Nuclear transfection was performed by using the Nucleofector system (Amaxa Biosystems, Koln, Germany). Cells $\left(1 \times 10^{5}\right)$ were suspended in $150 \mu \mathrm{l}$ Nucleofector solution (Amaxa Biosystems) containing $100 \mathrm{nM}$ miR-34b precursor, antisense miR-34b inhibitor or respective controls at room temperature. Each assay was performed in triplicate.

MTT assay. Transfected cells were seeded in 96-well plates at a density of $1 \times 10^{5}$ cells $/ 100 \mu \mathrm{l}$. Cell proliferation was measured using the MTT method. After $72 \mathrm{~h}, 20 \mu \mathrm{l}$ of $5 \mathrm{mg} /$ ml MTT solution was added to each well and plates were incubated for $3 \mathrm{~h}$ at $37^{\circ} \mathrm{C}$. Absorbance at $490 \mathrm{~nm}$ was determined using a SPECTRAmax 340 (Molecular Devices Corporation). Six wells were assayed for each set of conditions and stadard deviations (SDs) were determined.

Statistical analysis. Data are expressed as means \pm SD. Statistical analyses were performed using the software package StatView (Abacus Concepts, Berkeley, CA, USA). The

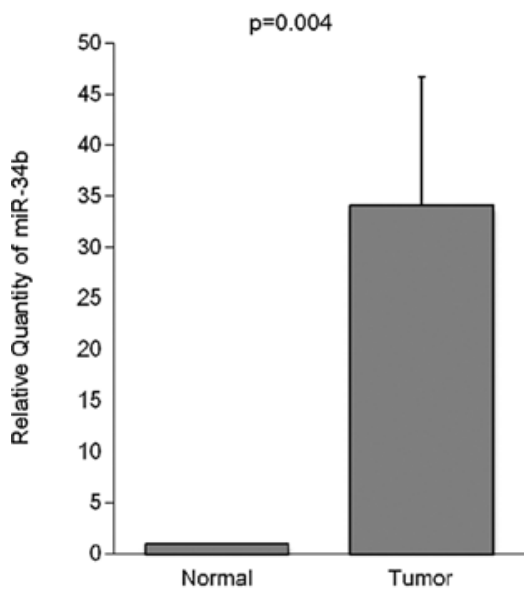

Figure 1. Comparison of miR-34b expression in ESCC tissue and noncancerous mucosa. miR-34b expression levels were significantly higher in ESCC tissue than in the corresponding noncancerous mucosa $(n=88, P=0.004)$.

Wilcoxon signed-rank, Mann-Whitney U and Kruskal-Wallis tests were used to evaluate the significance of differences in the expression levels of miR-34b. In all analyses, $\mathrm{P}<0.05$ was considered to be statistically significant.

\section{Results}

Expression of miR-34b in esophageal cancer tissue. Quantitative RT-PCR was used to evaluate the miRNA expression in 88 tumors and paired normal esophageal tissues. The expression level of miR-34b was significantly higher in the tumor tissue than in the corresponding non-cancerous mucosa (Table I). The expression of miR-34b in the normal esophageal mucosa was very low (Fig. 1).

The relationship between miR-34b expression, in 88 ESCC samples, and patient clinicopathological factors was examined (Table I). The miR-34b expression levels in patients with advanced stage 2, 3 or 4 were higher than those in patients with stage 0 or 1 tumors $(\mathrm{P}=0.039$, Mann-Whitney $\mathrm{U}$ test $)$ (Fig. 2). No significant differences were noted in miR-34b expression with respect to age, gender, the depth of invasion (T-factor), lymph node status, histological differentiation, lymphatic invasion, blood vessel invasion and p53 IHC. Additionally, no significant relationship was found between miR-34b expression and patient survival (data not shown).

Expression of miR-34b in esophageal cancer cell lines. The expression of miR-34b was investigated in 15 esophageal cancer cell lines (TE1-15) and 1 non-cancerous esophageal squamous epithelial cell line (Het-1A) using quantative RT-PCR. The expression of miR-34b and U6B was detectable in all of the cell lines studied. The level of expression of miR-34b varied among the cell lines: TE2 had the lowest expression and TE8 the highest (Fig. 3).

Effects of suppression of miR-34b in ESCC cell lines. To examine the role of miR-34b on the proliferation of ESCC cells, antisense miRNA inhibitor or respective controls were transfected into TE8 cells (the highest expressor of miR-34b among the TE series cell lines). MTT assay confirmed that 
Table I. Correlation of miR-34b expression with clinicopathological factors in esophageal cancer.

\begin{tabular}{lcc}
\hline Characteristics & No. of patients $(\mathrm{n}=88)$ & miR-34b expression \\
\hline Normal & 88 & 1.00 \\
Tumor & 88 & $34.18 \pm 12.55$ \\
Age at surgery & & \\
$\leq 65$ years & 48 & $20.89 \pm 9.61$ \\
$>65$ years & 40 & $50.12 \pm 25.04$ \\
Gender & & \\
Male & 72 & $39.96 \pm 15.26$ \\
Female & 16 & $8.14 \pm 2.85$ \\
Tumor status & & \\
T1 & 21 & $7.41 \pm 3.08$ \\
T2 & 12 & $32.44 \pm 21.09$ \\
T3 & 32 & $59.68 \pm 29.71$ \\
T4 & 23 & $24.04 \pm 21.26$ \\
T1 vs. T2, -3, -4 & & \\
T1, -2 vs. T3, -4 & & \\
T1, -2, -3 vs. T4 & &
\end{tabular}

Lymph node status

$\begin{array}{lr}\text { N0 } & 24 \\ \text { N1 } & 14 \\ \text { N2 } & 27 \\ \text { N3 } & 12 \\ \text { N4 } & 9 \\ \text { Unknown } & 2\end{array}$

$44.63 \pm 33.69$

$33.81 \pm 29.25$

$10.07 \pm 3.84$

$4.43 \pm 2.07$

$85.04 \pm 58.27$

N0 vs. N1, -2, -3

$186.29 \pm 185.93$

Pathological stage

0

I

5

$7.51 \pm 4.43$

$1.64 \pm 0.73$

II

10

$66.67 \pm 47.07$

III 26

$23.59 \pm 15.96$

IV

$40.23 \pm 21.46$

0, I vs. II, III, IV

Histological differentiation

Well

Moderate

Poor

$13.30 \pm 5.30$

Well vs. moderate, poor

Well, moderate vs. poor

Lymphatic invasion

Negative $\quad 17$

$4.77 \pm 2.02$

Positive

$35.92 \pm 13.43$

Unknown

Blood vessel invasion

$\begin{array}{lr}\text { Negative } & 29 \\ \text { Positive } & 45 \\ \text { Unknown } & 14 \\ \text { p53 IHC } & \\ \text { Negative } & 23 \\ \text { Positive } & 36 \\ \text { Unknown } & 29\end{array}$




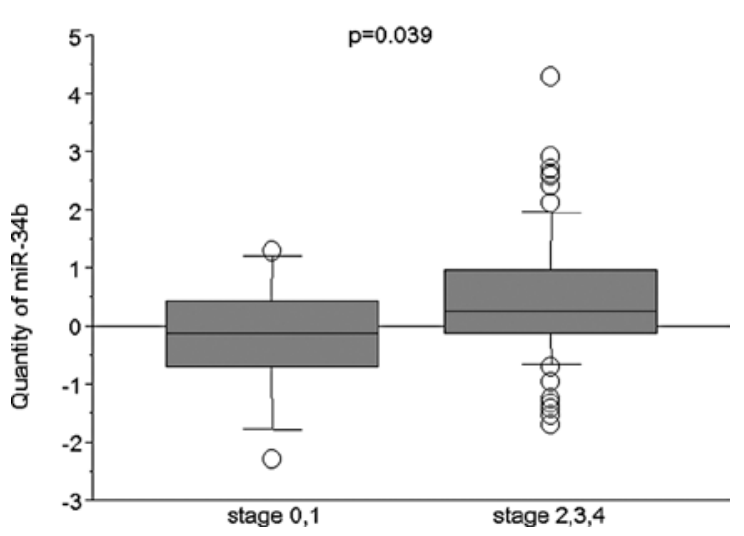

Figure 2. Comparison of miR-34b expression in stage 0 and 1 , as well as more advanced stage 2, 3 and 4 ESCC. miR-34b expression levels were higher in patients with more advanced stage 2, 3 and 4 than in those with stage 0 and 1 tumors $(\mathrm{P}=0.039)$.

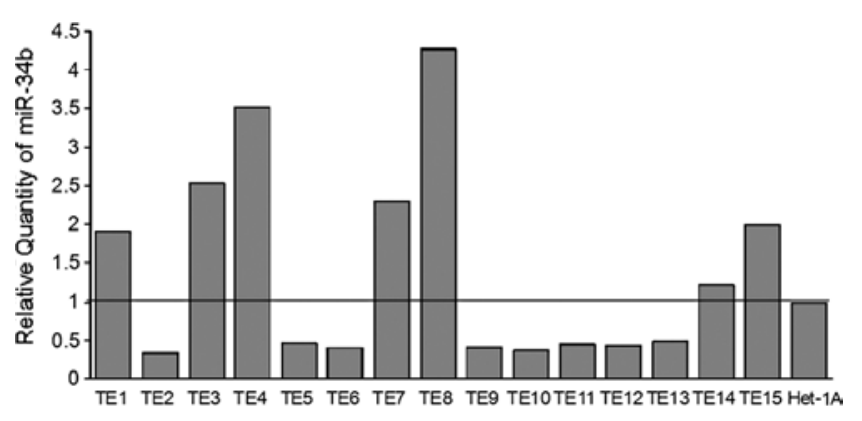

Figure 3. miR-34b expression in esophageal cancer cell lines. TE2 had the lowest and TE8 the highest expression of miR-34b among the cell lines studied as compared to Het-1A.

the proliferation of anti-miR-34b-transfected TE1, TE3, TE8 and TE15 cells was significantly lower as compared to control inhibitor-transfected cells on Day 3 (Fig. 4). When TE6, a miR-34b-low cell line, was used no difference was noted in the proliferation following the transfection of antisense miR-34b (data not shown). Anti-miR miRNA inhibitors (patent pending) are designed to bind to and inhibit not the expression of miR-34b, but the activity of endogenous miRNAs when introduced into cells.

Effects of overexpression of miR-34b in TE6 and TE9 cells. To determine whether the overexpression of miR-34b affects the proliferation of TE6 and -9 cells, the miRNA precursor was transfected into these cells, which express miR-34b at low levels. The expression levels of miR-34b in pre-miR-34btransfected cells were higher than control precursor miR-transfected cells. However, no significant difference was observed in the proliferation of these cells as compared to cells transfected with control precursor miR on Day 3 (Fig. 5).

\section{Discussion}

We analyzed the expression of miR-34b in 88 ESCC samples by RT-PCR. The expression levels of miR-34b in ESCC were significantly higher than those in normal esophageal tissues. Moreover, miR-34b was more highly expressed in tumors
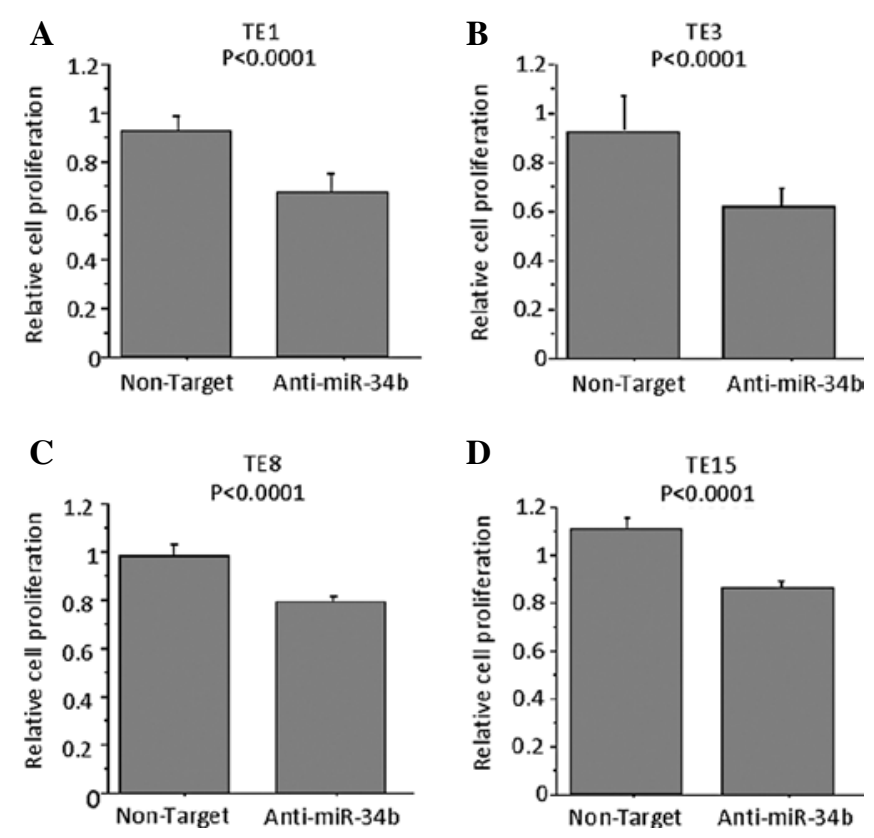

Figure 4. Effects of transfection with anti-miR-34b in TE1, $-3,-8$ and -15 cells. (A) Cell proliferation of anti-miR-34b- or control inhibitor-transfected TE1 cells. TE1 cell proliferation rates were determined by MTT assay on Day 3 and absorbance values were determined on a SPECTRAmax 340 at $490 \mathrm{~nm}$. Proliferation of anti-miR-34b-transfected TE1 cells as lower compared to control inhibitor-transfected cells. (B) Proliferation of anti-miR-34b-transfected TE3 cells were lower as compared to control inhibitor-transfected cells in the same way. Proliferation rates determined for (C) TE8 and (D) TE15 were as in (A).
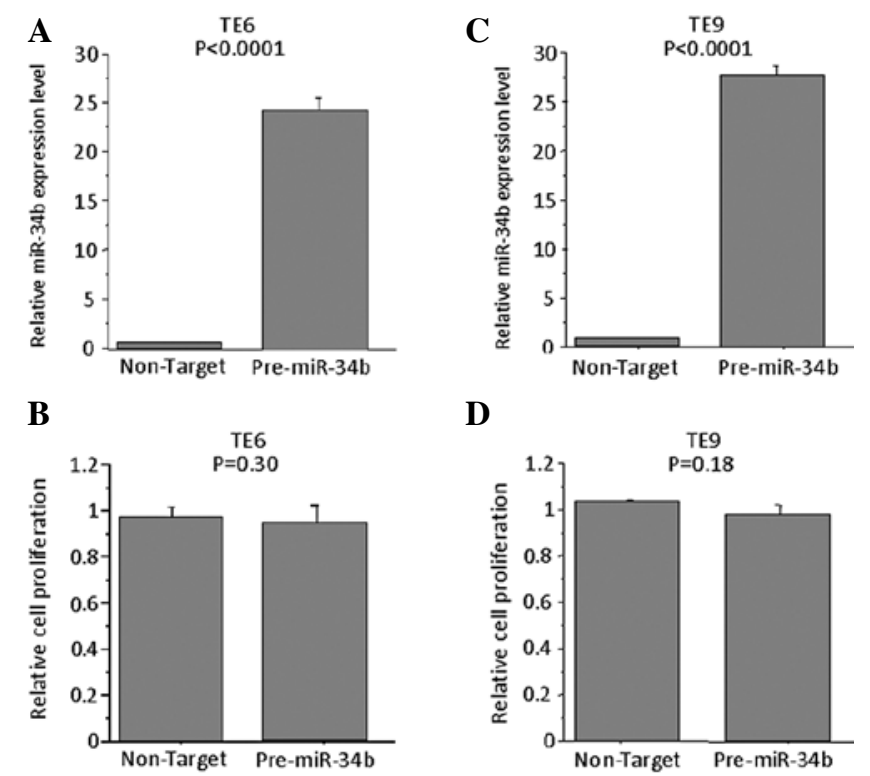

Figure 5. Effects of transfection with pre-miR-34b in TE6 and -9 cells. (A) TE6 cells were transiently transfected with $100 \mathrm{nM}$ pre-miR-34b or control precursor. This effect was examined by quantitative RT-PCR on Day 3. (B) Cell proliferation of pre-miR-34b- or control precursor-transfected in TE6 cells. TE6 cell proliferation rates were determined by MTT assay on Day 3 and absorbance values were determined on a SPECTRAmax 340 at $490 \mathrm{~nm}$. No significant difference was noted in the proliferation of TE6 cells as compared to those trasfected with control precursor miR. Proliferation rates determined for (C) TE9 were as in (A), and (D) TE9 as in (B).

with stages 2-4 than in those with stages 0 or 1 . These results suggest that the expression of miR-34b is oncogenic in ESCC. 
miR-34b is located at chromosome 11q23.1. Recently, 11q23 amplification was described as a new cytogenetic entity in myeloid malignancies (9). However, no study is currently available on the amplification of 11q23.1 in ESCC.

It was reported that the expression of miR-34b is high in squamous cell carcinoma of the tongue (10) and undifferentiated gastric cancer (11). To study the functional roles of miR-34b in ESCC, we knocked down miR-34b using the specific inhibitor in the ESCC cell lines. We found that the proliferation of ESCC cell lines with a high expression of miR-34b was inhibited by antisense miR-34b. The results indicate a close association between miR-34b expression and the proliferation of ESCC cells. However, not all of the ESCC cell lines expressed miR-34b at higher levels than the normal esophageal cell line (Het-1A) (Fig. 3). Additionally, the overexpression of miR-34b in the miR-34b-low TE6 or -9 cell lines had no effect on proliferation. These results indicate that the role of miR-34b in cell proliferation is limited to subsets of ESCC patients.

miR-34b was recently identified as a p53 target and a potential tumor suppressor (12-15). Over 50\% of human cancers have mutant p53 and the expression of miR-34a, b and c appears to be correlated with p53 $(16,17)$. In human tumors, the selective pressure to lose miR-34s may be relieved by the frequent mutation of p53. Thus, genetic alterations in miR-34s are more likely to occur in tumor types that characteristically contain wild-type p53 (18). The mutation of p53 was found in approximately $50 \%$ of cell lines and the primary tumor of the esophagus (19). In our study, p53 immunohistochemistry was positive in $59.3 \%$ of the ESCC patients. However, no difference was found in the expression of miR-34b between the p53-positive and -negative patients. Our study suggests that miR-34b functions as an oncogene as opposed to a tumor suppressor. It is possible that miR-34b functions in a context-dependent manner or that other factors may regulate miR-34b.

In esophageal cancer, it has been reported that a high expression of miR-103/miR-107 is associated with a poor prognosis (20). miRNA processing enzyme (RNASEN) was elevated in a proportion of ESCC and a high RNASEN expression correlates with poor prognosis in ESCC (21). Thus, it appears that miRs play a role in ESCC.

In conclusion, further studies on the role of miR-34b on ESCC progression including identification of its target genes are warranted.

\section{Acknowledgements}

The authors would like to thank Ms. Shinobu Makino for the excellent technical assistance.

\section{References}

1. McManus MT: MicroRNAs and cancer. Semin Cancer Biol 13: 253-258, 2003.

2. Michael MZ, O' Connor SM, van Holst Pellekaan NG, Young GP and James RJ: Reduced accumulation of specific microRNAs in colorectal neoplasia. Mol Cancer Res 12: 882-891, 2003.

3. Calin GA and Croce CM: MicroRNA-cancer connection: the beginning of a new tale. Cancer Res 66: 7390-7394, 2006.

4. Esquela-Kerscher A and Slack FJ: Oncomirs - microRNAs with a role in cancer. Nat Rev Cancer 6: 259-269, 2006.

5. Meng F, Henson R, Wehbe-Janek H, Ghoshal K, Jacob ST and Patel T: MicroRNA-21 regulates expression of the PTEN tumor suppressor gene in human hepatocellular cancer. Gastroenterology 133: 647-658, 2007.

6. Lu J, Getz G, Miska EA, et al: MicroRNA expression profiles classify human cancers. Nature 435: 834-838, 2005.

7. Ogawa R, Ishiguro H, Kuwabara $\mathrm{Y}$, et al: Expression profiling of microRNAs in human esophageal squamous cell carcinoma using RT-PCR. Med Mol Morphol 42: 102-109, 2009.

8. Livak KJ and Schmittgen TD: Analysis of relative gene expression data using real-time quantitative PCR and the 2(-Delta Delta C(T)) Method. Methods 25: 402-408, 2001.

9. Poppe B, Vandesompele J, Schoch, C, et al: Expression analyses identify MLL as a prominent target of 11q23 amplification and support an etiologic role for MLL gain function in myeloid malignancies. Blood 103: 229-235, 2004.

10. Wong TS, Liu XB, Wong BY, Ng RW, Yuen AP and Wei WI: Mature miR-184 as potential oncogenic microRNA of squamous cell carcinoma of tongue. Clin Cancer Res 14: 2588-2592, 2008.

11. Katada $\mathrm{T}$, Ishiguro $\mathrm{H}$, Kuwabara $\mathrm{Y}$, et al: microRNA expression profile in undifferentiated gastric cancer. Int $\mathbf{J}$ Oncol 34: 537-542, 2009.

12. Chang TC, Wentzel EA, Kent OA, et al: Transactivation of miR-34a by 53 broadly influences gene expression and promotes apoptosis. Mol Cell 26: 745-752, 2007.

13. He L, He X, Lim LP, et al: A microRNA component of the p53 tumour suppressor network. Nature 447: 1130-1134, 2007.

14. Hermeking H: p53 enters the microRNA world. Cancer Cell 12: 414-418, 2007.

15. Raver-Shapira N, Marciano E, Meiri E, et al: Transcriptional activation of miR-34a contributes to p53-mediated apoptosis. Mol Cell 26: 731-743, 2007.

16. Bommer GT, Gerin I, Feng Y, et al: p53-mediated activation of miRNA34 candidate tumor-suppressor genes. Curr Biol 17: 1298-1307, 2007.

17. He $\mathrm{X}, \mathrm{He} \mathrm{L}$ and Hannon GJ: The guardian's little helper: microRNAs in the p53 tumor suppressor network. Cancer Res 67: 11099-11101, 2007.

18. Tarasov V, Jung P, Verdoodt B, et al: Differential regulation of microRNAs by p53 revealed by massively parallel sequencing: miR-34a is a p53 target that induces apoptosis and G1-arrest. Cell Cycle 6: 1586-1593, 2007.

19. Nishihira T, Hashimoto Y, Katayama M, Mori S and Kuroki T: Molecular and cellular features of esophageal cancer cells. J Cancer Res Clin Oncol 119: 441-449, 1993.

20. Guo Y, Chen Z, Zhang L, et al: Distinctive microRNA profiles relating to patient survival in esophageal squamous cell carcinoma. Cancer Res 68: 26-33, 2008.

21. Sugito N, Ishiguro H, Kuwabara Y, et al: RNASEN regulates cell proliferation and affects survival in esophageal cancer patients. Clin Cancer Res 12: 7322-7328, 2006. 